\section{II-06 ANTIBODY CROSS-LINKING OF CD14 ACTIVATES MERTK AND PROMOTES HUMAN MACROPHAGE PHAGOCYTOSIS OF APOPTOTIC NEUTROPHILS: THE DUAL ROLE OF CD14 AT THE CROSSROADS BETWEEN M1 AND M2C POLARIZATION}

Gaetano Zizzo, Philip L Cohen*. Section of Rheumatology, Lewis Katz School of Medicine at Temple University, Philadelphia, PA, USA

10.1136/lupus-2018-Ism.105

Background Mer receptor tyrosine kinase (MerTK) is key for efficient phagocytosis of apoptotic neutrophils (ANs) and homeostasis of IL-10 production by human anti-inflammatory M2c monocytes/macrophages. We asked whether stimulation of certain M2c surface receptors contribute in turn to MerTK activation.

Methods Human monocytes/macrophages were differentiated under M1, M2a and M2c polarizing conditions. We tested the effects of antibody-mediated cross-linking of M2c receptors (i. e., CD14, CD16, CD32, CD163, CD204) on MerTK phosphorylation and phagocytosis of ANs. MerTK expression was also studied by flow cytometry and western blot in the presence of LPS and in M2c-derived microvesicles (MVs).

Results Antibody cross-linking of either CD14 or CD32/Fc $\gamma$ RII led to Syk activation and MerTK phosphorylation in its two distinct glycoforms (175-205 and 135-155 KDa). Cross-linked CD14 enhanced efferocytosis by M2c macrophages and enabled M1 and M2a cells to clear ANs efficiently. In M1 conditions, LPS abolished surface MerTK expression on CD14 ${ }^{\text {bright }}$ cell subsets, so disrupting the anti-inflammatory pathway. In M2c cells, instead, MerTK was diffusely and brightly co-expressed with CD14, and was also detected in M2c macrophage-derived MVs; in these conditions, LPS only partially down-regulated MerTK on the cell surface, while the smaller MerTK glycoform contained in MVs remained intact.

Conclusions Cooperation between CD14 and MerTK fosters tethering and engulfment of ANs by human monocytes/macrophages. CD14 stands between M1-related LPS co-receptor activity and M2c-related MerTK-dependent responses. MerTK interaction with CD32/Fc $\gamma \mathrm{RII}$, its detection in M2c MVs, and the differential localization and LPS susceptibility of MerTK glycoforms add further new elements to the complexity of the MerTK network.

Acknowledgements NIAID and Lupus Research Alliance

\section{II-07 PATHOGENESIS OF PULMONARY LUPUS AND VASCULITIS: TRAFFICKING OF INNATE B1 B CELLS TO THE LUNGS AS A NOVEL MECHANISM}

\footnotetext{
1,2,3,4 Ram R Singh* 1,2Priti Prasad, ${ }^{3}$ Michael Fishbein, ${ }^{1}$ Isela Valera. 'Autoimmunity and Tolerance Laboratory, Division of Rheumatology, Department of Medicine; ${ }^{2}$ Molecular Toxicology Interdepartmental Program; ${ }^{3}$ Department of Pathology and Laboratory Medicine; ${ }^{4}$ Jonsson Comprehensive Cancer Center, David Geffen School of Medicine at University of California Los Angeles, USA
}

10.1136/lupus-2018-Ism.106

Background Up to 50\% patients with SLE may develop pneumonitis, vasculitis and diffuse pulmonary hemorrhage (DPH). Advances in the pathogenesis of pulmonary lupus have been hampered because of the heterogeneity of clinical findings and paucity of access to the affected tissue. Hydrocarbon oils, such as 2,6,10,14-tetramethylpentadecane (TMPD), induce lupus-like autoantibodies, nephritis, arthritis, pneumonitis, and
DPH depending on the animals' genetic background. Humans can be exposed to hydrocarbon oils in crude oil and in mineral oils used in cosmetics, laxatives, and food-coatings. Here, we used this model to investigate the pathogenesis of pulmonary lupus, focusing on the role of innate B1 B-cells.

Methods We injected TMPD intraperitoneally in C57BL/6 (B6) mice to induce pulmonary lupus. We adoptively transferred wild-type peritoneal fluid cells which are enriched in B1 Bcells into CD19 ${ }^{-/-}$(that have less B1a B-cells) and in $\operatorname{Ig}^{-/-}$ mice (have no B-cells), and tracked the transferred cells using CD45.1/CD45.2 system. Effect on disease was assessed using weight-loss, a semiquantitative scoring, and a quantitative measurement for lung hemorrhage. We analyzed global gene expression in the lungs using Affymatrix microarray.

Results In wild-type B6 mice, TMPD injection caused weightloss, pneumonitis, vasculitis, and/or DPH in $73 \%$ of 62 animals compared to none of control animals injected with control hydrocarbon oil hexadecane or with PBS or sham. Immunophenotyping revealed abnormalities of all immune cells tested in the diseased lungs. At earlier timepoints prior to histopathological changes, while both hexadecane and TMPD caused myeloid cell abnormalities, only TMPD caused lunginfiltration with B-cells that expressed B1 B-cell subset markers: $\mathrm{CD} 19^{+} \mathrm{CD} 11 \mathrm{~b}^{+} / \mathrm{CD} 19^{+} \mathrm{CD}^{+}$. Such B1 B-cells were simultaneously reduced in their usual location (peritoneal cavity). CD $19^{-/-}$mice that have less B1a B-cells developed less $\mathrm{DPH}$, and less B-cell infiltration in the lungs than wildtype mice. The adoptive transfer of wildtype peritoneal fluid cells into the peritoneum of $\mathrm{CD} 19^{-/-}$or $\operatorname{Ig}^{-/-}$mice induced more $\mathrm{DPH} /$ pneumonitis than the respective knockout recipients reconstituted with $\mathrm{CD} 19^{-/}$B-cells. The adoptive transfer of CD $45.1^{+}$wildtype peritoneal fluid cells into the peritoneum of $\mathrm{CD} 45.2^{+} \mathrm{CD} 19^{-/-}$recipients led to lung-infiltration with CD 45. $1^{+}$B-cells. Furthermore, TMPD induced in the lungs a differential expression of $\mathrm{Cxcl13}$ that is known to drive B1 Bcells' migration.

Conclusions Exposure to TMPD induces B1 B-cells to traffic from the peritoneum into the lungs and cause pneumonitis/ DPH. Identification of this mechanism in human lupus will have important implications for targeting a specific B-cell subset as a potential therapy.

\section{II-08 COMPLEMENT RECEPTORS 1 AND 2 ON B CELL SUBSETS ARE NEGATIVELY ASSOCIATED WITH LUPUS DISEASE ACTIVITY}

Shoshana Rosenthal, Dominik Reinhold, Kenneth L Jones, Weiming Zhang, Duane Pearson, Kara M Lough, Susan A Boackle*. University of Colorado School of Medicine, Aurora, CO

\subsection{6/lupus-2018-Ism.107}

Background B cell complement receptor 1 (CR1) and complement receptor 2 (CR2) levels are decreased by $\sim 50 \%$ in subjects with systemic lupus erythematosus (SLE). CR2 but not CR1 levels have been negatively correlated with lupus disease activity. However, increased CR1 expression on B cells is associated with a protective polymorphism in the CR2 gene. We conducted a longitudinal analysis of $\mathrm{B}$ cell CR1 and CR2 to further evaluate an association with lupus disease activity and to determine whether it is driven by specific cell subsets.

Methods Thirty-six subjects meeting the revised 1982 ACR criteria for SLE were enrolled. Each subject had a baseline 
visit and 1 to 4 follow up visits, with additional visits for flares. Peripheral blood mononuclear cells (PBMC) were stained with a dead cell marker and antibodies to CD19, CD27, and IgD. Antibody binding capacity (ABC) of CR1 and CR2 on live bulk and gated B cells was determined using quantitative flow cytometry (Bang Labs). The frequency of live gated B cells was determined. Disease activity was measured using SLEDAI. Statistical analyses were performed using R. To determine associations between the SLEDAI scores and gate/quadrant signals, negative binomial mixed effect models were fitted using the glmmADMB package. A random effect intercept was included to account for different individual baseline SLEDAI scores. The gate/ quadrant signals, in terms of $\mathrm{ABC}$ and percentages, were the independent variables. Adjustments were made for age, prednisone dose, and, where relevant, bead lot. To account for multiple testing in bulk B cells, a conservative Bonferroni adjustment was used, whereas in gated cells, the p-values were adjusted to control the false discovery rate according to Benjamini-Hochberg. A p-value of $\leq 0.05$ was considered significant.

Results CR1 and CR2 ABC on bulk B cells and on $\mathrm{IgD}+\mathrm{CD} 27-, \operatorname{IgD}+\mathrm{CD} 27+$, and $\operatorname{IgD}-/ \mathrm{CD} 27$ +gated $\mathrm{B}$ cells was negatively associated with lupus disease activity, whereas there was no association between CR1 and CR2 $\mathrm{ABC}$ on IgD-/CD27- B cells or frequency of any of these gated populations and disease activity (table 1 and data not shown).

\begin{tabular}{|c|c|c|c|c|}
\hline B cells & Independent variable & Estimate & Standard Error & Adjusted $p$ value \\
\hline Bulk & CR1 ABC & $-3.07 E-05$ & $1.09 \mathrm{E}-05$ & 0.018771099 \\
\hline Bulk & CR2 ABC & $-3.49 \mathrm{E}-05$ & $9.77 \mathrm{E}-06$ & 0.001413636 \\
\hline $\lg D+C D 27-$ & CR1 $A B C$ & $-1.47 \mathrm{E}-05$ & $6.50 \mathrm{E}-06$ & 0.031872055 \\
\hline $\lg D+C D 27-$ & $C R 2 A B C$ & $-3.06 \mathrm{E}-05$ & $9.65 \mathrm{E}-06$ & 0.005642294 \\
\hline $\lg \mathrm{D}+\mathrm{CD} 27+$ & CR1 ABC & $-1.74 \mathrm{E}-05$ & $6.32 \mathrm{E}-06$ & 0.013441797 \\
\hline $\lg \mathrm{D}+\mathrm{CD} 27+$ & CR2 ABC & $-2.46 \mathrm{E}-05$ & $7.88 \mathrm{E}-06$ & 0.005642294 \\
\hline $\operatorname{lgD}-\mathrm{CD} 27+$ & CR1 ABC & $-2.77 \mathrm{E}-05$ & $8.63 \mathrm{E}-06$ & 0.005642294 \\
\hline IgD-CD27+ & CR2 ABC & $-4.25 \mathrm{E}-05$ & $1.21 \mathrm{E}-05$ & 0.003641553 \\
\hline IgD-CD27- & CR1 ABC & $-2.53 \mathrm{E}-07$ & $2.48 \mathrm{E}-06$ & 0.918673439 \\
\hline $\operatorname{lgD}-C D 27-$ & CR2 ABC & $-3.72 \mathrm{E}-06$ & $1.10 \mathrm{E}-05$ & 0.785689588 \\
\hline
\end{tabular}

Conclusions Both CR1 and CR2 levels are negatively associated with lupus disease activity in defined B cell subsets. Although this study does not prove causality, these data suggest that altered levels of these receptors on specific B cell subsets may predict disease flare or be associated with disease remission.
Acknowledgements This work was supported by R01AI070304, K24AI078004, and the Lupus Research Institute.

\section{II-09 IMMUNOLOGIC PROPERTIES OF CUTANEOUS LUPUS ERYTHEMATOSUS (CLE) PATIENTS REFRACTORY TO ANTIMALARIALS COMPARED TO PATIENTS THAT RESPOND TO ANTIMALARIALS}

${ }^{1,2}$ Krisha Desai, ${ }^{1,2}$ Majid Zeidi, ${ }^{1,2,3}$ Hee Joo Kim, ${ }^{1,2}$ Victoria P Werth* ${ }^{*}{ }^{1}$ Corporal Michael J.
Crescenz VAMC, Philadelphia, PA, USA; ${ }^{2}$ Department of Dermatology, University of
Pennsylvania, Philadelphia, PA, USA; ${ }^{3}$ Department of Dermatology, Gil Medical Center,
Gachon University College of Medicine, Incheon, Korea

\subsection{6/lupus-2018-Ism.108}

Background Two major therapies for cutaneous lupus erythematosus (CLE) are the antimalarials, hydroxychloroquine (HCQ) and quinacrine (QC). HCQ is often the first-line therapy for CLE, but only half of patients show a response to it. While some of the patients that do not initially respond to HCQ benefit from the addition of QC, there is a subset of patients that are refractory to both antimalarials. Refractoriness poses a huge challenge because these patients will often continue to have active disease when they are initially started on antimalarials. To better characterize these refractory patients, we investigated the immunologic characteristics of patients that respond to antimalarials versus those that do not.

Methods CLE patients were classified as HCQ-responders, HCQ/QC-responders, or HCQ/QC-nonresponders. Immunohistochemistry was used to characterize the inflammatory cell composition and cytokine expression in lesional skin biopsies from patients. Total RNA was extracted from these biopsies to analyze specific gene signatures. The patient's CLASI score - a measure of disease activity - at the time of the biopsy was also determined.

Results Immunohistochemistry showed that myeloid dendritic cells (mDCs) were significantly higher in HCQ/QC-responders compared to HCQ-responders and HCQ/QC-nonresponders, while plasmacytoid dendritic cells, neutrophils, macrophages, and autoreactive $\mathrm{T}$ cells did not differ significantly among the three groups. The HCQ/QC-nonresponder group was distinct from the other groups in that their CLASI scores did correlate positively with the number of macrophages $(p<0.05$, figure $1)$. Staining also showed that IL-22 expression was significantly higher in HCQ/QC nonresponders versus the HCQ or HCQ/QC- responders while IL-17 expression was not significantly different between the responders and nonresponders. Analyzing the mRNA expression demonstrated a high type I IFN signature (LY6E, OAS1, ISG15, MX1) in HCQ-
A

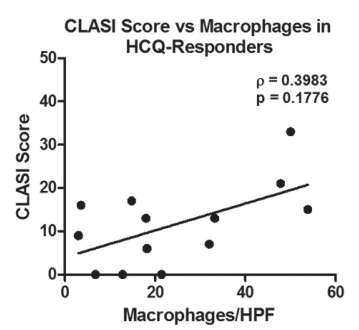

B

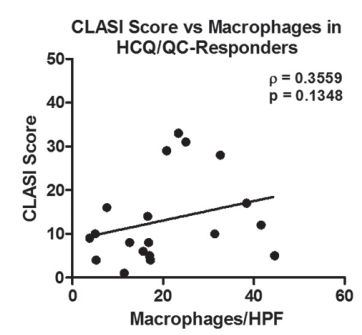

C

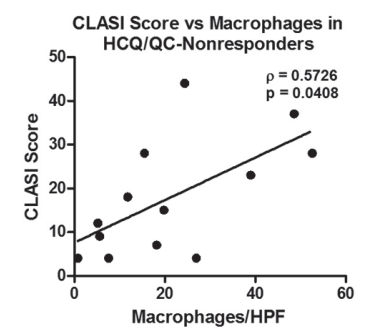

Abstract II-09 Figure 1 